\title{
Effect of replacing the general energy-coupling proteins of the PEP: sugar phosphotransferase system of Salmonella typhimurium with their fructose-inducible counterparts on utilization of the PTS sugar glucitol
}

\author{
Sarah L. Sutrina, Lisa Alleyne, Keisher Hoyte and Margot Blenman
}

Author for correspondence: Sarah L. Sutrina. Tel: +1 246417 4360. Fax: +1 2464174325. e-mail: ssutrina@uwichill.edu.bb

Department of Biological and Chemical Sciences, The University of the West Indies, Cave Hill Campus, Barbados

\begin{abstract}
A strain of Salmonella typhimurium in which the genes encoding the general phosphoenolpyruvate:sugar phosphotransferase system (PTS) proteins HPr and Enzyme I have been deleted, the normally cryptic gene encoding the fructoseinducible Enzyme I (EI* or El fructose) is expressed, and the fructose repressor protein is inactive (fruR or cra mutant) was studied. This strain lacks HPr and EI, but expresses FPr (DTP) and EI fructose constitutively. Since FPr and EI ${ }^{\text {fructose }}$ can substitute for HPr and El, the strain grew in minimal liquid medium supplemented with the PTS sugars glucose, fructose, $\mathbf{N}$-acetylglucosamine, mannitol or mannose. However, it showed very poor to negligible growth on the PTS sugar glucitol. It also grew very poorly on the non-PTS sugars maltose, melibiose and especially glycerol. Adding cAMP to the medium allowed growth on glucitol, but did not affect growth on glycerol. We suggest that poor phosphorylation of the regulatory molecule Enzyme IIAglucose by FPr is responsible for these effects.
\end{abstract}

Keywords: phosphoenolpyruvate: sugar phosphotransferase system, carbon catabolite control, gene regulation, carbohydrate transport

\section{INTRODUCTION}

The bacterial phosphoenolpyruvate (PEP): sugar phosphotransferase system (PTS) catalyses the uptake and concomitant phosphorylation of a number of carbohydrates (for reviews see Postma et al., 1993; Meadow et al., 1990; Saier, 1989). In Escherichia coli and Salmonella typhimurium, PTS sugars include glucose, mannose, fructose, mannitol, glucitol, galactitol and $\mathrm{N}$-acetylglucosamine. The general energy-coupling proteins Enzyme I (EI) and $\mathrm{HPr}$ interact with sugar-specific Enzymes II (EII $\left.{ }^{\text {sugar }}\right)$, with the exception of the fructose system, which has its own HPr-like domain, FPr. EI autophosphorylates, using PEP as the phosphate donor; the phosphoryl group is then transferred successively to $\mathrm{HPr}$, then to the A domain of a sugar-specific EII, then to the B domain of the same EII, then to the incoming

\footnotetext{
Abbreviations: CAP, catabolite activator protein; DTP, diphosphoryltransfer protein; EI/EII, enzymes I/II; Fru, fructose; Glc, glucose; GICNAc, Nacetylglucosamine; Gut, glucitol; Mtl, mannitol; PEP, phosphoenolpyruvate; PTS, phosphotransferase system.
}

sugar as it enters the cell via the C domain of the EII. Other carbohydrates are taken up by other mechanisms. These non-PTS sugars include melibiose, glycerol and maltose in both E. coli and S. typhimurium, as well as lactose in E. coli.

The fructose PTS is structurally unique in that its HPr counterpart is a domain of a larger protein, DTP (diphosphoryltransfer protein); the other two domains are the sugar-specific phosphocarrier domain EIIA ${ }^{\text {Fru }}$ and a third domain $M$ (modulator) which is thought to play a regulatory role (Geerse et al., 1989; Wu et al., 1990). It has been long established that FPr can substitute for HPr in PTSs for other sugars besides fructose both in vivo and in vitro (Saier et al., 1970, 1976). In complementation assays it was observed that purified $\mathrm{FPr}$, whether as part of the larger DTP or in the form of a $9 \mathrm{kDa}$ separate protein (free FPr), worked about $5 \%$ as well as $\mathrm{HPr}$ in the PTSs for mannose, $\mathrm{N}$-acetylglucosamine, mannitol and glucitol, but only $0.5 \%$ as well as HPr in the glucose-specific PTS (Sutrina et al., 1988). More recently, in vitro assays by a different group indicated that both DTP and free FPr accepted a phosphate group 
from EI about $40 \%$ as well as $\mathrm{HPr}$, and donated the phosphate group to EIIA ${ }^{\mathrm{Mtl}}$ about as well as HPr (Lux et al., 1995). These results taken together suggest that FPr, molecule for molecule, does not interact with EI as effectively as $\mathrm{HPr}$, and that it also interacts relatively poorly with the A domain of the glucose-specific Enzyme II $\left(\right.$ EIIA $\left.^{\mathrm{Glc}}\right)$ compared to other EIIAs. In a recent study, DTP was also shown to substitute for HPr in both the activation of the antiterminator BglG of the $\beta$-glucosides $(b g l)$ operon of E. coli and in the negative regulation of this same protein by phospho-Enzyme IIBCA ${ }^{\text {Bgl }}$ (Görke \& Rak, 1999).

In E. coli and S. typhimurium, EIIA ${ }^{\text {Gle }}$ mediates control of utilization of non-PTS sugars by the PTS (reviewed by Saier, 1989; Postma et al., 1993; Meadow et al., 1990). A model to account for this was proposed. When in its phosphorylated form, EIIA ${ }^{\text {Gle }}$-P activates adenylate cyclase (Reddy \& Kamireddi, 1998). When in its unphosphorylated form, EIIA ${ }^{\text {Glc }}$ prevents the uptake of non-PTS sugars by binding to and inhibiting the permeases for these sugars, or, in the case of glycerol, by binding to and inhibiting glycerol kinase, a phenomenon called inducer exclusion. In the absence of PTS sugars, the ratio of EIIA ${ }^{\text {Gle }}$ P to EIIA ${ }^{\text {Glc }}$ is relatively high, leading to relatively high levels of cAMP and to active non-PTS permeases. This allows for high level expression of catabolite activator protein (CAP)-cAMPactivated operons in general and to induction of the genes for the uptake and utilization of the non-PTS sugars in particular. In the presence of a PTS sugar, phosphate is drained off EIIA ${ }^{\mathrm{Glc}}$-P, and inducer exclusion/catabolite repression results. Recent studies challenge the model just described; it has been proposed, based on these studies, that the role of cAMP in glucose/lactose diauxie in E. coli is to enhance inducer exclusion by activating the membrane component of the glucose PTS, EIICB ${ }^{\mathrm{Glc}}$, at the transcriptional level (Inada et al., 1996; Kimata et al., 1997). In contrast, another recent study suggests that, in E. coli, cAMP-dependent catabolite repression, rather than inducer exclusion, may be the dominant mechanism by which glucose represses glycerol kinase levels during diauxic growth (Holtman et al., 2001). In S. typhimurium, both mechanisms may be important in glucose/glycerol diauxie (Novotny et al., 1985).

In S. typhimurium strain LJ705 the structural genes for EI and HPr have been deleted, and the fruR gene encoding the fructose repressor protein FruR, or Cra, has been disrupted by a Tn10 insertion; this strain also expresses the normally cryptic EI-like protein $\mathrm{EI}^{\mathrm{Fru}}$ (EI*), which is part of the fru regulon (Chin et al., 1987). This strain is able to grow on PTS sugars, since the constitutively expressed $\mathrm{FPr}(\mathrm{DTP})$ and $\mathrm{EI}^{\mathrm{Fru}}$ substitute for the missing HPr and EI. In this study, we found that growth of LJ705 on the PTS sugar glucitol as its sole carbon source is poor to negligible and that its growth on the non-PTS sugars melibiose, maltose and especially glycerol is also poor. Addition of cAMP to the growth medium markedly improved growth on glucitol, but not on glycerol. We propose that underphosphorylation of
EIIA $^{\text {Gle }}$ by FPr in this strain is responsible for these effects; low cAMP leads to low activation of the CAP-cAMP-dependent glucitol operon (Yamada \& Saier, 1988) and a high EIIA ${ }^{\text {Glc }} /$ EIIA $^{\text {Gle }}$-P ratio causes inducer exclusion of non-PTS sugars even in the absence of PTS sugars. In this study we have focussed primarily on the lack of ability of LJ705 to grow on glucitol. Its poor growth on non-PTS sugars is currently under further investigation.

\section{METHODS}

Growth rate studies. Bacterial strains were grown in either Luria-Bertani (LB) broth or minimal medium 63 (Saier et al., 1970) supplemented with tryptophan $\left(50 \mu \mathrm{g} \mathrm{ml}^{-1}\right)$ and carbohydrates (at concentrations indicated). Other supplements and antibiotics were included where indicated. Strains were incubated at $37^{\circ} \mathrm{C}$, at $175-180$ r.p.m. Growth was monitored by measuring the optical density at $540 \mathrm{~nm}$, using a Beckman DU-65 spectrophotometer with a $1 \mathrm{~cm}$ cuvette.

Strains. Salmonella typhimurium strains used were LJ705 ( $\Delta c y s K$ ptsHI41 fruR51::Tn10 ptsJ52), SB1475 (ptsH15), SB3507 (trpB223), SB1667 (mal) and SB1744 (mtlA61). The ptsJ52 mutation in LJ705 allows expression of the normally cryptic EI ${ }^{\mathrm{Fru}}$. All of these strains are derivatives of wild-type strain LT-2. Escherichia coli strains used were K12 (wild-type) and ESK147 $\left(\mathrm{F}^{-}\right.$ompT $\mathrm{r} \beta-\mathrm{m} \beta-$ plys $\left.S \mathrm{~cm}^{\mathrm{r}}\right)$. Strains ESK147 (E. B. Waygood, Department of Biochemistry, University of Saskatchewan), SB3507 (Cordaro \& Roseman, 1972) and SB1667 (M. H. Saier, Jr, University of California at San Diego) are wild-type with respect to the PTS.

Preparation of crude extracts. Cultures were grown to late exponential phase in LB broth and harvested by centrifugation. The pellets were washed twice with $0 \cdot 15 \mathrm{M} \mathrm{NaCl}$ and once with buffer $(50 \mathrm{mM}$ Tris/ $\mathrm{HCl}, \mathrm{pH} 7 \cdot 5,1 \mathrm{mM}$ EDTA, $0.1 \mathrm{mM}$ PMSF, $0.2 \mathrm{mM}$ DTT), then resuspended in a small volume of the same buffer. The suspensions were passed through the French press three times at 10000 p.s.i. (69 MPa). Unbroken cells and debris were removed by centrifugation (10000 r.p.m., $10 \mathrm{~min}$, JA17 rotor).

Preparation of washed membranes. Crude extracts were subjected to centrifugation for $4 \mathrm{~h}$ at 15000 r.p.m. in a JA17 rotor. The membrane pellets were resuspended in the above buffer and the centrifugation step was repeated. The washed membrane pellets were resuspended in a minimal volume of buffer.

Assays. Complementation assays for PTS components were conducted as previously described, using ${ }^{14} \mathrm{C}$-labelled sugar substrates and PEP (Reizer et al., 1992). In assays of crude extracts for the sugar-specific PTS components, partially purified HPr and EI were added to ensure an excess of these general energy-coupling proteins.

Transphosphorylation assays for the glucitol-specific membrane component EII(BC) $)^{\text {Gut }}$ were conducted as previously described (Saier et al., 1977). Assay mixtures contained $10 \mu \mathrm{M}$

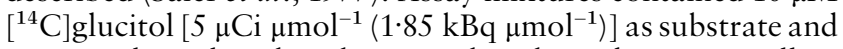
$10 \mathrm{mM}$ glucitol 6-phosphate as phosphate donor as well as $50 \mathrm{mM} \mathrm{KPO}_{4}$, pH 6.0, $25 \mathrm{mM} \mathrm{KF}, 12.5 \mathrm{mM} \mathrm{MgCl}_{2}, 2.5 \mathrm{mM}$ DTT and the membrane preparation being assayed; the total volume was $100 \mu \mathrm{l}$. After incubation for $30 \mathrm{~min}$ at $37^{\circ} \mathrm{C}$, the reactions were stopped by adding $1 \mathrm{ml}$ water. The $\left[{ }^{14} \mathrm{C}\right]$ glucitol phosphate product was collected using anion exchange columns and quantified with a scintillation counter (Kundig \& 
Roseman, 1971). The Lowry assay was used to determine total protein concentration.

\section{RESULTS AND DISCUSSION}

\section{Growth of LJ705 versus wild-type strains}

S. typhimurium strain LJ705 lacks the general PTS component proteins $\mathrm{HPr}$ and EI but produces their normally fructose-inducible counterparts $\mathrm{FPr}$ and $\mathrm{EI}^{\mathrm{Fru}}$ constitutively. The latter proteins substitute for the former, allowing the strain to utilize PTS sugars (Chin et al., 1987). We compared growth of this strain under various conditions to that of two wild-type S. typhimurium strains, SB3507 and SB1667, and two wild-type E. coli strains, ESK147 and K12.

On minimal medium, growth of LJ705 was poor to negligible, with respect to both rate and extent, on the PTS sugar glucitol relative to its growth on other PTS sugars (glucose, fructose, $\mathrm{N}$-acetylglucosamine, mannose and mannitol) or galactose. Growth on mannitol was also somewhat slow compared to the other PTS sugars, and growth on glucose (and galactose, which is not shown) somewhat fast (Fig. 1b). The growth rate did not increase when the concentration of glucitol was increased from the $0 \cdot 2 \%$ used in most of our studies to $0.5 \%$ or $1 \%$; decreasing the concentration also did not promote growth. This strain also grew very poorly on the non-PTS sugars maltose, melibiose and especially glycerol (not shown). We normally included tetracycline $\left(20 \mu \mathrm{g} \mathrm{ml}^{-1}\right)$ in the medium for LJ705; omission of the drug did not affect the growth rate.

The wild-type S. typhimurium strain SB3507 grew faster than LJ705 on all the PTS sugars; it also grew more slowly, and with an initial lag, on glucitol than on other PTS sugars, but was clearly able to utilize glucitol (Fig. 1a). Both of the wild-type E. coli strains also grew on glucitol, as did another wild-type $S$. typhimurium strain, SB1667. Like SB3507, strain SB1667 grew relatively slowly (with a similar lag period) on glucitol relative to other PTS sugars.

On rich medium, little difference in growth rate or extent was observed when LJ705 was grown on unsupplemented LB or on glucose, fructose, mannitol or glucitol or combinations of glucitol and one of the other sugars, and there was no large difference between growth rates of LJ705 and the wild-type strain SB3507. Supplementing minimal medium with tryptone $(0.5 \%)$ also minimized the differences in growth rate. On
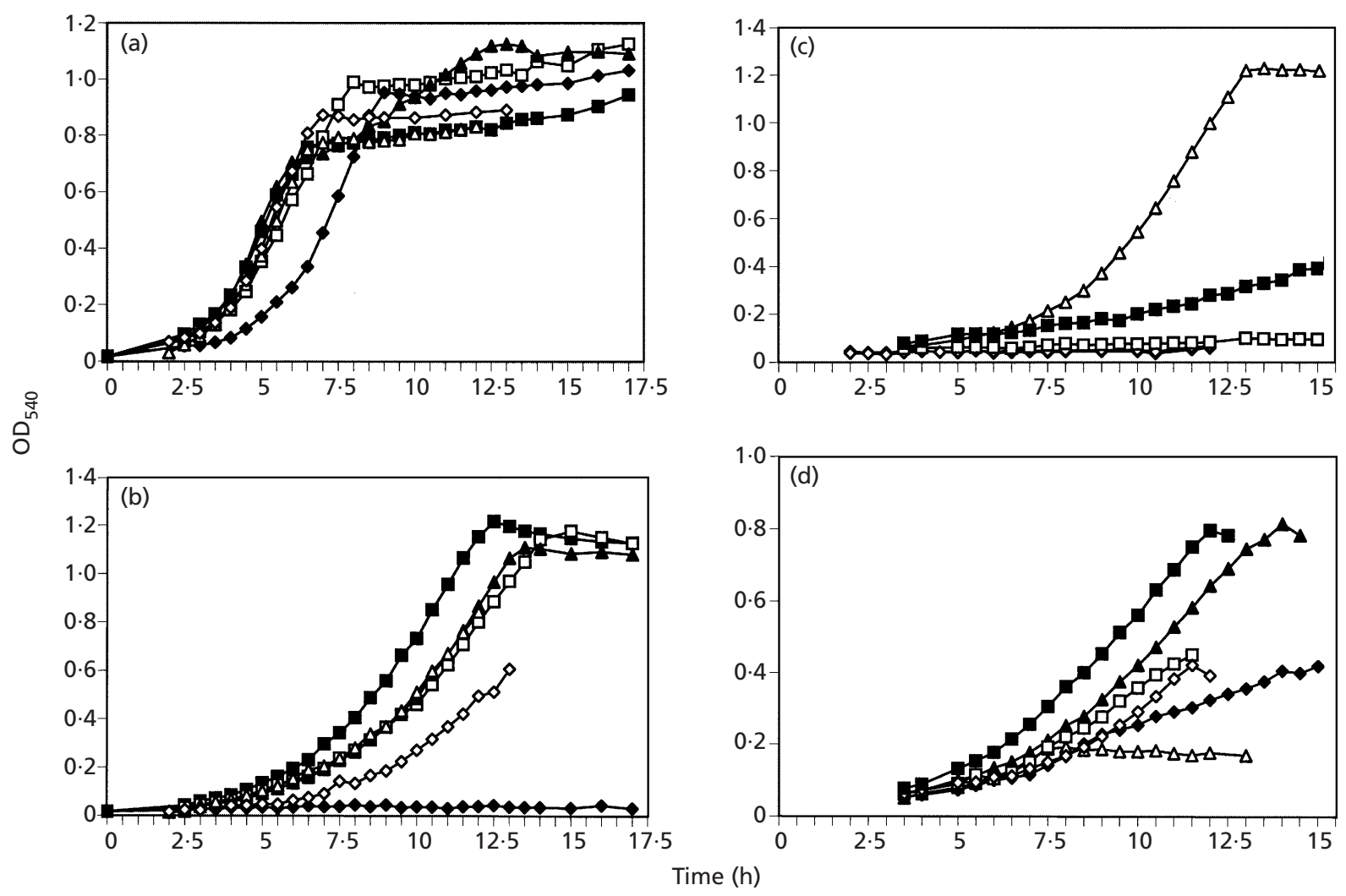

Fig. 1. Growth of wild-type (SB3507), LJ705 and HPr mutant on PTS sugars. Strains of S. typhimurium were grown in minimal medium supplemented with $50 \mu \mathrm{g}$ tryptophan $\mathrm{ml}^{-1}$ and $0.2 \%$ of the indicated carbohydrate. (a) SB3507 (wildtype), (b) LJ705, (c) SB1475 (ptsH). $\square$, Glucose; $\square$, mannose; $\boldsymbol{\Delta}, N$-acetylglucosamine; $\diamond$, glucitol; $\diamond$, mannitol; $\triangle$, fructose. (d) SB1475 was grown on $0.02 \%$ fructose $(\triangle)$ or on mixtures of $0.02 \%$ fructose and $0.2 \%$ of the indicated carbohydrate. $\boldsymbol{\square}$, glucose; $\square$, mannose; $\boldsymbol{\Delta}, N$-acetylglucosamine; $\diamond$ glucitol; $\diamond$, mannitol. 
fermentation plates, glucitol effects were subtle; LJ705 generally showed a slightly slower positive response on EMB or MacConkey plates containing glucitol than did wild-type strains. This was not reported previously. All strains tested showed a weak response on glucitol plates relative to other PTS sugars.

Since glucitol did not hinder growth of LJ705 on rich medium or on medium supplemented with other sugars, the effect did not appear to be due to toxicity due, for example, to build-up of glucitol 6-phosphate as a result of defective glucitol-6-phosphate dehydrogenase. Rather, the strain seemed to be unable to utilize glucitol as a growth substrate.

\section{Growth of a ptsH mutant versus LJ705}

One possible explanation for the inability of LJ705 to grow on glucitol is that $\mathrm{FPr}$ interacts so poorly with the glucitol-specific PTS protein EIIA ${ }^{\text {Gut }}$ that glucitol cannot be taken up fast enough to support growth. This seemed unlikely since a previous in vitro study indicated that FPr substitutes equally well for HPr in the PTSs for mannitol, mannose, glucitol and $\mathrm{N}$-acetylglucosamine, and relatively $(10 \times)$ poorly only in the glucose system (Sutrina et al., 1988). On the other hand, the in vitro study was done under conditions such that crossreactivity between the glucitol and mannitol systems could have confused the results (Saier et al., 1977). To investigate this possibility, we compared growth of LJ705 to that of S. typhimurium strain SB1475 (Saier et al., 1976), which carries a point mutation in the structural gene for $\mathrm{HPr}, p t s H$. The latter strain has negligible HPr activity, and because the $p t s H$ mutation is pleiotropic, it also has low EI activity; in contrast to LJ705, it does not express $\mathrm{EI}^{\mathrm{Fru}}$ and production of $\mathrm{FPr}$ (DTP) is not constitutive.

As expected, SB1475 grew relatively well (usually slightly more slowly than LJ705) on fructose, but not on most other PTS sugars, as the sole carbon source; it grew, slowly, on glucose (Fig. 1c). As expected, on minimal medium supplemented with a mixture of fructose $(0.02 \%)$ and glucitol, mannitol, mannose or $\mathrm{N}$ acetylglucosamine $(0 \cdot 2 \%)$, this strain continued to grow on the other PTS sugar after the fructose should have been consumed (or grew on both sugars simultaneously), using fructose-induced FPr to substitute for HPr in the uptake of the other PTS sugars (Fig. 1d). Thus, poor interaction of FPr with the glucitol PTS cannot be the sole explanation for the failure of LJ705 to grow on glucitol. Interestingly, glucitol and mannitol seemed to slow down growth of SB1475 on fructose, $\mathrm{N}$-acetylglucosamine may have increased the growth rate on fructose slightly, and growth on a mixture of fructose and glucose was monophasic and considerably more rapid than on fructose alone. The latter result was surprising, since FPr works poorly with EIIA ${ }^{\text {Gle }}$, but was consistent with the finding that LJ705 grew better on glucose than on other PTS sugars. The fact that glucose is capable of entering S. typhimurium cells via routes other than the glucose-specific PTS, e.g., it is a good substrate of the mannose PTS and is also a substrate of the non-PTS galactose permease (Postma \& Stock, 1980), probably accounts for these results.

\section{Effect of CAMP on growth of LJ705}

Although FPr substitutes for HPr, it interacts relatively poorly with EI (Lux et al., 1995; Sutrina et al., 1988), and, as mentioned above, the latter study suggested that, relative to other EIIAs, its interaction with EIIA ${ }^{\text {Gle }}$ is particularly poor. Thus this mediator of inducer exclusion/catabolite repression may be underphosphorylated in LJ705. It is possible that the substitution of $\mathrm{EI}^{\mathrm{Fru}}$ for EI also contributes to this effect; in vitro studies suggested that $\mathrm{EI}^{\mathrm{Fru}}$ behaves very similarly to EI, but these studies were not extensive (Chin et al., 1987). This protein remains something of a mystery, since it has not been identified as any of the known paralogues of EI in the sequences of E. coli or S. typhimurium. Since the phosphorylated form of EIIA Glc mediates catabolite repression by activating adenylate cyclase, its underphosphorylation, by FPr, in LJ705 could lead to low levels of cAMP, and thus to low levels of expression of cAMP-dependent operons. The gut operon absolutely requires cAMP for expression (Yamada \& Saier, 1988). The non-PTS operons for glycerol, melibiose and maltose utilization are also under CAP-cAMP control.

To test our hypothesis, we added $1 \mathrm{mM}$ cAMP to the medium of either LJ705 or the wild-type strain SB3507 growing on $0 \cdot 2 \%$ glucitol. The cAMP had little effect on the growth rate of the wild-type strain (Fig. 2a). The growth rate of LJ705 increased markedly, after a lag; when the experiment was extended further than the one shown in Fig. 2(b), the final density of the culture approached that of the wild-type strain. We also added cAMP to cultures of the wild-type strain, LJ705 and the mutant SB1475 growing on a mixture of $0 \cdot 2 \%$ glucitol and $0.02 \%$ fructose. The wild-type strain kept growing on glucitol after the fructose should have been exhausted (or grew on both sugars simultaneously; there was no obvious lag phase); cAMP had no effect on the growth rate (Fig. 2a). After exhaustion of fructose, the growth rate of L J705 dropped to its usual negligible level; cAMP allowed a continuation of growth on glucitol, after a lag, and a final density approaching that of the wild-type culture (Fig. 2b). As above, glucitol appeared to slow down the growth of SB1475 on fructose, but growth continued, at a slow rate, after the fructose should have been exhausted. Addition of cAMP did not appear to affect the growth rate of this mutant strongly; in the experiment shown, a slight positive effect was observed (Fig. 2c), while a duplicate experiment showed a slight negative effect.

The experiment was repeated with cultures of SB3507 and LJ705 growing on minimal medium supplemented with $0.2 \%$ glycerol or with a mixture of $0.2 \%$ glycerol and $0.02 \%$ fructose (not shown). The wild-type strain grew somewhat slowly on glycerol compared to glucose, with an initial lag; LJ705 grew slowly after a very long initial lag $(8-12 \mathrm{~h})$. On the mixture, the wild-type strain 

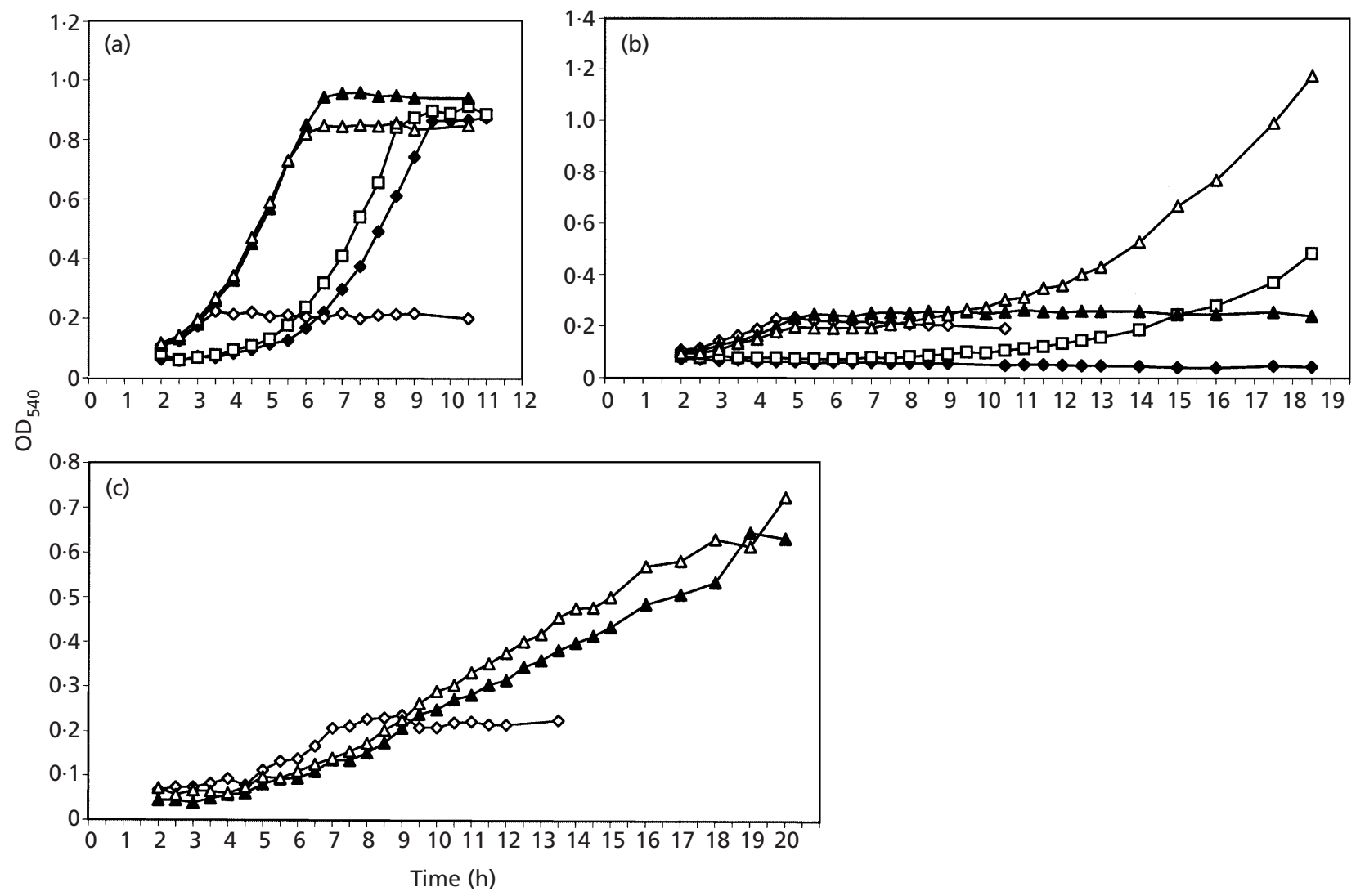

Fig. 2. Effect of CAMP on growth of wild-type (SB3507), LJ705 and HPr mutant on glucitol. S. typhimurium strains were grown in minimal medium supplemented with $50 \mu \mathrm{g}$ tryptophan $\mathrm{ml}^{-1}$ and $0.02 \%$ fructose or $0.2 \%$ glucitol, or a mixture of $0.02 \%$ fructose and $0.2 \%$ glucitol. Where indicated, $1 \mathrm{mM}$ CAMP was added at $2 \mathrm{~h}$. (a) SB3507 (wild-type), (b) LJ705, (c) SB1475. $\diamond$, Fructose; $\diamond$, glucitol; $\square$, glucitol + cAMP; $\mathbf{\Delta}$, fructose + glucitol; $\triangle$, fructose + glucitol + cAMP.

Table 1. Specific activities of glucitol-specific Enzyme II(BC) in membrane preparations

All units are pmol $\left[{ }^{14} \mathrm{C}\right]$ glucitol 6-phosphate $\min ^{-1}(\mathrm{mg} \text { protein })^{-1}$. Where standard deviations are given, values represent the average of $2-5$ independent membrane preparations. ND, Not determined.

\begin{tabular}{|c|c|c|c|c|c|}
\hline \multirow[t]{2}{*}{ Growth conditions* } & \multicolumn{5}{|c|}{ Strain } \\
\hline & SB3507 (WT) & LJ705 & SB1475 $\left(\mathrm{HPr}^{-}\right)$ & SB1677 (WT) & $\mathrm{SB} 1744(m t l A)$ \\
\hline No sugar & $16 \cdot 2 \pm 4 \cdot 8$ & $8 \cdot 2$ & $25 \cdot 6 \pm 12 \cdot 5$ & $79 \cdot 4$ & $31 \cdot 8$ \\
\hline Gut & $370 \pm 59$ & $160 \pm 19$ & $52 \cdot 9 \pm 15 \cdot 1$ & 427 & 232 \\
\hline Gut + cAMP & $539 \pm 132$ & $315 \pm 31$ & $\mathrm{ND}$ & ND & ND \\
\hline Gut + Glc & $129 \pm 18 \cdot 1$ & $77 \cdot 3 \pm 2 \cdot 9$ & ND & ND & ND \\
\hline $\mathrm{Gut}+\mathrm{Glc}+\mathrm{cAMP}$ & 100 & 108 & ND & ND & ND \\
\hline Gut $+\mathrm{Mtl}$ & $22 \cdot 0 \pm 3 \cdot 6$ & $18 \cdot 1 \pm 9 \cdot 1$ & ND & ND & 176 \\
\hline $\mathrm{Gut}+\mathrm{Mtl}+\mathrm{cAMP}$ & $22 \cdot 4$ & $15 \cdot 5$ & ND & ND & ND \\
\hline Gut + Fru & $278 \pm 56$ & $66 \cdot 7 \pm 8 \cdot 6$ & $161 \pm 24$ & ND & ND \\
\hline Gut + low Fru & 390 & 183 & 155 & ND & ND \\
\hline Gut + GlcNAc & 117 & $36 \cdot 7$ & ND & ND & ND \\
\hline Glc & $32 \cdot 4$ & $24 \cdot 5$ & ND & ND & $\mathrm{ND}$ \\
\hline Mtl & $21 \cdot 6$ & $25 \cdot 5$ & ND & ND & $30 \cdot 6$ \\
\hline Fru & $29 \cdot 5$ & $14 \cdot 6$ & $17 \cdot 5$ & ND & $\mathrm{ND}$ \\
\hline GlcNAc & $27 \cdot 5$ & $2 \cdot 55$ & ND & ND & ND \\
\hline
\end{tabular}

* $0.5 \%$ sugar, except 'low fructose' (0.02\%). Gut, glucitol; Glc, glucose; Mtl, mannitol; Fru, fructose; GlcNAc, N-acetylglucosamine. 

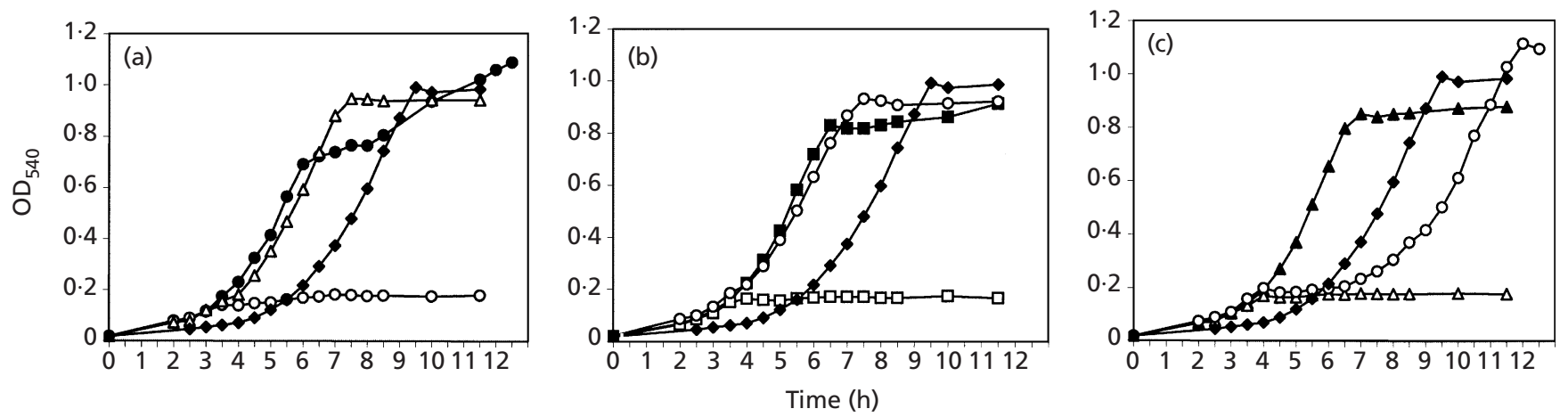

Fig. 3. Growth of wild-type (SB3507) on mixtures of glucitol and other PTS sugars. S. typhimurium strain SB3507 was grown on minimal medium supplemented with $50 \mu \mathrm{g}$ tryptophan $\mathrm{ml}^{-1}$ and $0.2 \% \mathrm{~N}$-acetylglucosamine, glucose, mannitol or glucitol, and with mixtures of $0.02 \%$ of each of the other sugars and $0.2 \%$ glucitol, as indicated. (a) 0, $0.2 \%$ GlcNAc; $0,0.02 \%$ GlcNAc; $\triangle$, GlcNAc/glucitol; $\bullet, 0.2 \%$ glucitol. (b) $\mathbf{\square}, 0.2 \%$ glucose; $\square, 0.02 \%$ glucose; $\bigcirc$, glucose/glucitol; $\bullet$, $0.2 \%$ glucitol. (c) $\Delta, 0.2 \%$ mannitol; $\triangle, 0.02 \%$ mannitol; $\bigcirc$, mannitol/glucitol; $\bullet, 0.2 \%$ glucitol.

continued to grow on glycerol after the fructose should have been exhausted, while LJ705 showed a long lag before resuming slow growth on glycerol. Addition of cAMP during the lag period had negligible effect on the growth rate of LJ705 on glycerol or on the mixture of fructose and glycerol.

The results suggest that low expression of the gut operon due to low levels of cAMP is responsible for poor growth of LJ705 on glucitol. On glycerol, addition of cAMP should promote catabolite derepression, but a relatively high ratio of unphosphorylated EIIA ${ }^{\text {Glc }}$ to EIIA $^{\mathrm{Glc}}-\mathrm{P}$ may still cause inducer exclusion (EIIA ${ }^{\text {Gle }}$ inhibits glycerol kinase). Our results suggest that the gut operon of S. typhimurium requires higher levels of cAMP for full activation than do the man, $m t l, g l c$ and nag PTS operons.

\section{Levels of glucitol-specific PTS components in LJ705 versus other strains}

To determine the levels of the glucitol-specific PTS components, strains of $S$. typhimurium were grown in liquid LB broth supplemented with $0.5 \%$ glucitol or with $0.5 \%$ glucitol plus another PTS sugar (usually $0.5 \%)$ and/or cAMP $(1 \mathrm{mM}$, added at $2.5 \mathrm{~h})$; crude extracts were then prepared and assayed as previously described (Reizer et al., 1992). The results (not shown) were difficult to interpret due to interference by the mannitol-specific PTS component EIICBA ${ }^{\mathrm{Mtl}}$ in the glucitol PTS assay (Grenier et al., 1985; Saier et al., 1977). To eliminate this problem, washed membranes were prepared and the transphosphorylation assay was carried out using $\left[{ }^{14} \mathrm{C}\right]$ glucitol as substrate and glucitol 6-phosphate as phosphate donor (Saier et al., 1977). Results, expressed as pmol $\left[{ }^{14} \mathrm{C}\right]$ glucitol 6-P formed $\min ^{-1}$ (mg membrane protein) ${ }^{-1}$, are shown in Table 1. The specific activity of the membrane component of the glucitol PTS, EII (BC) ${ }^{\text {Gut }}$, was consistently lower (by $50-70 \%$ ) in membranes from glucitol-grown LJ705 compared to membranes from glucitol-grown wild-type strain SB3507. Another wild-type strain, SB1667, was also found to have high specific activity relative to LJ705. In the presence of $1 \mathrm{mM}$ cAMP, specific activities of EII(BC) ${ }^{\text {Gut }}$ in both SB3507 and LJ705 membranes increased, but the increase was more substantial for the mutant strain; values for the mutant strain grown with cAMP approached those for the wild-type strain grown without. Thus the rather small deficiency could be responsible for the inability of LJ705 to grow on glucitol.

Membrane preparations from SB1475 were also assayed for EIIA(BC) $)^{\text {Gut }}$. Maximal induction by glucitol was seen only in the presence of both glucitol and fructose. Membranes from cells grown in the presence of $0.5 \%$ glucitol and either $0.5 \%$ or $0.02 \%$ fructose showed EII(BC) $)^{\text {Gut }}$ specific activities similar to glucitol-grown LJ705. Thus it is not clear why this strain but not LJ705 continued to grow on glucitol after fructose should have been exhausted when exposed to a mixture of the two sugars.

Other PTS sugars appeared to repress expression of the glucitol PTS in the wild-type strain; interestingly, mannitol repressed most strongly, fructose least, and glucose and $\mathrm{N}$-acetylglucosamine repressed to a similar intermediate extent. Addition of cAMP did not relieve repression by glucose or mannitol. In strain SB1744 (Saier et al., 1976), which has a defective mannitol PTS, mannitol did not strongly repress expression of the glucitol PTS. Growth of wild-type strain SB3507 in the presence of PTS sugars other than glucitol generally resulted in a level of the membrane component EII(BC) $)^{\text {Gut }}$ about twice that of membranes from cells grown on unsupplemented LB, while glucitol induced about $20 \times$. Results were similar for LJ705, although repression by fructose appeared to be stronger than in the wild-type strain. Fructose, normally a Class B, or weakly repressing, PTS sugar, may be a Class A, or strongly repressing, PTS sugar in fruR (cra) strains (Crasnier-Mednansky et al., 1997). 


\section{Repression of the gut operon by mannitol in wild- type S. typhimurium}

To investigate these observations, further growth studies were conducted. When wild-type strain SB3507 was grown on a mixture of $0.02 \%$ mannitol and $0.2 \%$ glucitol, an obvious lag phase was observed between growth on mannitol and resumption of growth on glucitol (Fig. 3). Such a marked lag was not observed in the presence of $0.02 \%$ glucose, fructose or $\mathrm{N}$-acetylglucosamine and $0 \cdot 2 \%$ glucitol, and is consistent with strong repression of the glucitol operon in the presence of mannitol, and with early studies reporting diauxic growth of E. coli on mannitol and glucitol (Lengeler \& Lin, 1972).

\section{Conclusions}

A mutant strain (LJ705) of S. typhimurium in which the normally fructose-inducible (and, in the case of $\mathrm{EI}^{\mathrm{Fru}}$, normally cryptic) PTS proteins FPr (DTP) and EI ${ }^{\mathrm{Fru}}$ must substitute for the general PTS proteins HPr and EI showed poor to negligible growth on the PTS sugar glucitol as its sole carbon source. Since addition of cAMP to the medium allowed LJ705 to grow on glucitol, we hypothesized that low cAMP, which could result from underphosphorylation of the mediator of catabolite repression/inducer exclusion, EIIA ${ }^{\mathrm{Glc}}$, could be responsible. Determination of the levels of the glucitol PTS protein EII $(\mathrm{BC})^{\text {Gut }}$ in membranes from wild-type stains and LJ705 supported the hypothesis; induced levels in the mutant were consistently lower $(50-70 \%)$ than in the wild-type strains, and levels in the mutant grown with glucitol and cAMP in the medium approached those in the wild-type grown with glucitol but without cAMP.

Another possible explanation for the failure of LJ705 to grow on glucitol could be that FPr works too poorly with the glucitol-specific PTS proteins to support growth. To investigate this possibility, mutant strain SB1475, which lacks active HPr and has low EI activity but is wild-type with respect to the fructose-specific PTS, i.e., does not express $\mathrm{EI}^{\mathrm{Fru}}$ at all and does not express FPr (DTP) constitutively, was grown on a mixture of fructose and glucitol. This mutant was able to continue growing on the mixture to a density much higher than on fructose alone, suggesting that it must have been able to take up glucitol using FPr. Thus it seems unlikely that poor interaction of FPr with the glucitol PTS is the sole explanation for the poor growth of LJ705 on glucitol.

Membranes from SB1475 contained maximal levels of $\mathrm{EII}(\mathrm{BC})^{\text {Gut }}$ only when the strain was grown in the presence of both glucitol and fructose. Surprisingly, this maximal level was similar to that of LJ705 rather than to that of the wild-type strains. It should also be noted that SB1475 has been reported to produce levels of cAMP about $50 \%$ those of wild-type strains (Feldheim et al., 1990). Thus it is not clear why this strain was able to grow on glucitol, apparently using FPr, while LJ705 was not. Addition of cAMP to the medium did not strongly affect growth of SB1475 on a mixture of fructose and glucitol. Possibly relatively poor interaction of FPr and EIIA $^{\text {Gut }}$ is a contributing factor to the poor growth of LJ705 on glucitol, in addition to low EII(BC) Gut. It has been reported that increasing cAMP may lead to increased levels of fructose PTS proteins even in fruR strains (Crasnier-Mednansky et al., 1997). Growing LJ705 in the presence of cAMP could thus increase the levels of both FPr and the glucitol PTS components, as well as $\mathrm{EI}^{\mathrm{Fru}}$, and a combination of these effects may allow growth on glucitol.

\section{REFERENCES}

Chin, A. M., Sutrina, S., Feldheim, D. A. \& Saier, M. H., Jr (1987). Genetic expression of Enzyme I* activity of the phosphoenolpyruvate:sugar phosphotransferase system in ptsHI deletion strains of Salmonella typhimurium. J Bacteriol 169, 894-896.

Cordaro, J. C. \& Roseman, S. (1972). Deletion mapping of the genes coding for HPr and Enzyme I of the phosphoenolpyruvate:sugar phosphotransferase system in Salmonella typhimurium. J Bacteriol 112, 17-29.

Crasnier-Mednansky, M., Park, M. C., Studley, W. K. \& Saier, M. H., Jr (1997). Cra-mediated regulation of Escherichia coli adenylate cyclase. Microbiology 143, 785-792.

Feldheim, D. A., Chin, A. M., Nierva, C. T., Feucht, B. U., Cao, Y. W., Xu, Y. F., Sutrina, S. L. \& Saier, M. H., Jr (1990). Physiological consequences of the complete loss of phosphoryltransfer proteins HPr and FPr of the phosphoenolpyruvate: sugar phosphotransferase system and analysis of fructose (fru) operon expression in Salmonella typhimurium. J Bacteriol 172, 5459-5469.

Geerse, R. H., Izzo, F. \& Postma, P. W. (1989). The PEP: fructose phosphotransferase system in Salmonella typhimurium: FPr combines Enzyme III ${ }^{\text {fru }}$ and pseudo-HPr activities. Mol Gen Genet 216, 517-525.

Görke, B. \& Rak, B. (1999). Catabolite control of Escherichia coli regulatory protein BglG activity by antagonistically acting phosphorylations. EMBO J 18, 3370-3379.

Grenier, F. C., Hayward, I., Novotny, M. J., Leonard, J. E. \& Saier, M. H., Jr (1985). Identification of the phosphocarrier protein Enzyme III ${ }^{\text {glucitol }}$ : essential component of the glucitol phosphotransferase system in Salmonella typhimurium. J Bacteriol 161, 1017-1022.

Holtman, C. K., Pawlyk, A. C., Meadow, N. D. \& Pettigrew, D. W. (2001). Reverse genetics of Escherichia coli glycerol kinase allosteric regulation and glucose control of glycerol utilization in vivo. J Bacteriol 183, 3336-3344.

Inada, T., Kimata, K. \& Aiba, H. (1996). Mechanism responsible for glucose-lactose diauxie in Escherichia coli: challenge to the cAMP model. Genes Cells 1, 293-301.

Kimata, K., Takahashi, H., Inada, T., Postma, P. \& Aiba, H. (1997). cAMP receptor protein-cAMP plays a crucial role in glucoselactose diauxie by activating the major glucose transporter gene in Escherichia coli. Proc Natl Acad Sci U S A 92, 11583-11587.

Kundig, W. \& Roseman, S. (1971). Sugar transport. I. Isolation of a phosphotransferase system from Escherichia coli. J Biol Chem 246, 1393-1406.

Lengeler, J. \& Lin, E. C. C. (1972). Reversal of the mannitolsorbitol diauxie in Escherichia coli. J Bacteriol 112, 840-848.

Lux, R., Jahreis, K., Bettenbrock, K., Parkinson, J. S. \& Lengeler, J. W. (1995). Coupling the phosphotransferase system and the 
methyl-accepting chemotaxis protein-dependent chemotaxis signaling pathways of Escherichia coli. Proc Natl Acad Sci US A 92, 11583-11587.

Meadow, N. D., Fox, D. K. \& Roseman, S. (1990). The bacterial phosphoenolpyruvate:glycose phosphotransferase system. Annu Rev Biochem 59, 497-452.

Novotny, J. M., Frederickson, W. L., Waygood, E. B. \& Saier, M. H., Jr (1985). Allosteric regulation of glycerol kinase by enzyme III ${ }^{\text {glc }}$ of the phosphotransferase system in Escherichia coli and Salmonella typhimurium. J Bacteriol 162, 810-816.

Postma, P. W. \& Stock, J. B. (1980). Enzymes II of the phosphotransferase system do not catalyze transport in the absence of phosphorylation. J Bacteriol 141, 476-484.

Postma, P. W., Lengeler, J. W. \& Jacobson, G. R. (1993). Phosphoenolpyruvate: carbohydrate phosphotransferase systems of bacteria. Microbiol Rev 57, 543-594.

Reddy, P. \& Kamireddi, M. (1998). Modulation of Escherichia coli adenylyl cyclase activity by catalytic-site mutants of protein IIA ${ }^{\mathrm{Glc}}$ of the phosphoenolpyruvate:sugar phosphotransferase system. J Bacteriol 180, 732-737.

Reizer, J., Sutrina, S. L., Wu, L.-F., Deutscher, J., Reddy, P. \& Saier, M. H., Jr (1992). Functional interactions between proteins of the phosphoenolpyruvate:sugar phosphotransferase systems of $\mathrm{Ba}$ cillus subtilis and Escherichia coli. J Biol Chem 267, 9158-9169.

Saier, M. H., Jr (1989). Protein phosphorylation and allosteric control of inducer exclusion and catabolite repression by the bacterial phosphoenolpyruvate:sugar phosphotransferase system. Microbiol Rev 53, 109-120.
Saier, M. H., Jr, Simoni, R. D. \& Roseman, S. (1970). The physiological behavior of Enzyme I and heat-stable protein mutants of a bacterial phosphotransferase system. J Biol Chem 245, 5870-5873.

Saier, M. H., Jr, Simoni, R. D. \& Roseman, S. (1976). Sugar transport: properties of mutant bacteria defective in proteins of the phosphoenolpyruvate:sugar phosphotransferase system. J Biol Chem 251, 6584-6597.

Saier, M. H., Jr, Feucht, B. U. \& Mora, W. K. (1977). Sugar phosphate:sugar transphosphorylation and exchange group translocation catalyzed by the Enzyme II complexes of the bacterial phosphoenolpyruvate:sugar phosphotransferase system. J Biol Chem 252, 8899-8907.

Sutrina, S. L., Chin, A. M., Esch, F. \& Saier, M. H., Jr (1988). Purification and characterization of the fructose-inducible HPrlike protein, FPr, and the fructose-specific Enzyme III of the phosphoenolpyruvate:sugar phosphotransferase system of Salmonella typhimurium. J Biol Chem 263, 5061-5069.

Wu, L.-F., Tomich, J. M. \& Saier, M. H., Jr (1990). Structure and evolution of a multidomain multiphosphoryl transfer protein. J Mol Biol 213, 687-703.

Yamada, M. \& Saier, M. H., Jr (1988). Positive and negative regulators for glucitol (gut) operon expression in Escherichia coli. J Mol Biol 203, 569-583.

Received 15 April 2002; revised 23 August 2002; accepted 30 August 2002. 